\title{
BRIEF
}

\section{Use of MyDispense Among Pharmacy Programs Across the United States}

\author{
Chamipa Phanudulkitti, MBA, PhD, ${ }^{\mathrm{a}, \mathrm{b}}$ Clark Kebodeaux, PharmD, ${ }^{\mathrm{c}}$ Sarah E. Vordenberg, PharmD, $\mathrm{MPH}^{\mathrm{b}}$ \\ ${ }^{\text {a }}$ Burapha University, Faculty of Pharmaceutical Sciences, Chon Buri, Thailand \\ ${ }^{\mathrm{b}}$ University of Michigan, College of Pharmacy, Ann Arbor, Michigan \\ ${ }^{\mathrm{c}}$ University of Kentucky, College of Pharmacy, Lexington, Kentucky
}

Corresponding Author: Chamipa Phanudulkitti, Burapha University, Faculty of Pharmaceutical Sciences, 169 Long Had Bangsaen Rd, Saen Suk, Chon Buri District, Chon Buri 20131 Thailand. Tel: +66 84-665-4888. Email: chamipa@go.buu.ac.th

Submitted July 28, 2021; accepted January 2, 2022; ePublished January 2022

Objective. Pharmacy programs are increasingly using virtual simulation to provide a safe and realistic environment for students to practice critical patient care skills. The aim of this study was to describe the use of MyDispense, a virtual simulation tool, in pharmacy programs across the United States.

Methods. Researchers developed a 16-item survey focused on program characteristics and MyDispense integration in pharmacy curricula. It was sent to MyDispense administrators of pharmacy programs in the United States. Descriptive statistics were used to analyze the results.

Results. Administrators from 36 pharmacy programs responded to the survey (72\%). MyDispense was most commonly integrated into one or two courses for first- or second- year students. The most common skills that students practiced were medication dispensing, communication with patients, and drug-information skills. Validation exercises were most common followed by dispensing and over-the-counter exercises. The number of pharmacy schools using MyDispense suggest the potential for collaboration among instructors in order to increase efficiencies in delivery of course materials as well as evaluate student learning.

Conclusion. Instructors most commonly used MyDispense in courses for first- and second-year students to teach medication dispensing, communication with patients, utilizing drug information resources, applying pharmacy laws, and making recommendations about over-the-counter medications.

Keywords: simulation, pharmacy education, MyDispense

\section{INTRODUCTION}

Simulations play an essential role in educating health care professionals ${ }^{1}$ and allow learners to engage in a realistic situation or environment in order to safely develop knowledge and skills. ${ }^{2}$ Simulated learning environments can be applicable with large student numbers, enhance knowledge transfer to practical situations, and impact patient safety. ${ }^{3}$ Studies have demonstrated an increasing trend of simulation-based programs or virtual learning simulations used in various educational and training contexts to supplement traditional educational methods. ${ }^{4,5}$ Virtual simulation, in which students engage with a computer display simulating the physical world, provides an opportunity to increase knowledge, develop relevant skills, and increase learning motivation. ${ }^{5,6}$ In pharmacy education, simulations have been used in various areas such as pharmacotherapeutics, communication, manufacturing, law and ethics, and patient care and safety. ${ }^{7-11}$ Pharmacy programs are increasingly using simulation as it has been shown to positively impact problem solving in patient care topics, improve learner performance and satisfaction, and may advance the education and training for student pharmacists with the ultimate goal of enhancing patient care and safety.,6,9

MyDispense, a free web-based community pharmacy simulation program developed by Monash University, is designed as an authentic and safe learning and teaching environment to help student pharmacists develop their outpatient pharmacy skills and competency in dispensing medicinal products systematically, safely, and accurately without the danger of negative health outcomes. ${ }^{3,12,13}$ The program allows students the opportunity to engage with the entire process of dispensing while providing interactive explanations, self-assessment, and performance feedback. ${ }^{13,14}$ It has been used in both didactic and experiential curricula to teach topics such as medication dispensing, pharmacy skills, pharmacy law, and pharmacotherapeutics. ${ }^{8,9,15}$ McDowell et al. found that student pharmacists who used MyDispense in their dispensing tutorials reported positive perceptions of the tool to learn dispensing skills. ${ }^{3}$ Student pharmacists who completed MyDispense exercises in pharmacy law courses valued the innovative program and reported that the program helped them to recall pharmacy laws and focus on topics that were challenging. ${ }^{9,16}$ Mospan and Gillette also presented that there was a positive relationship between completion of MyDispense exercises and student pharmacists' performance on a pharmacy 
law exam scores. ${ }^{9}$ Shin et al. reported that exam scores were positively impacted when MyDispense was integrated into a therapeutics course. ${ }^{8}$ Instructors are able to develop customized dispensing, over-the-counter, and validation exercises that align with the complexity of the course or experience in which the content is integrated. ${ }^{17}$ Dispensing exercises focus on determining if a prescription is appropriate to dispense, and if so, to prepare the medication and provide patient counseling. Over-the-counter exercises focus on the provision of advice related to non-prescription medications. When completing validation exercises, the student checks the prescription, medication label, and medication bottle for accuracy, legality and medicine safety. ${ }^{12}$ The program is hierarchically organized in order to manage access to exercises and assessments. A unit can contain many tutorials and assessments; a tutorial can contain many exercises; assessments graded exercises. ${ }^{18}$ Several research teams reported positive performance and achievement of learning outcomes of student pharmacists who used MyDispense during their courses. ${ }^{9,15,19}$ Additionally, student pharmacists have reported positive perceptions about learning via this tool. ${ }^{9,20,21}$

After the original version of MyDispense was introduced by Monash University, Australia in 2010, it was expanded into different countries such as Namibia, South Africa, United States, Malaysia, Singapore, United Arab Emirates, and the United Kingdom. ${ }^{12}$ In 2014, the first regional version of MyDispense, the USA version, was released with support from Monash and five pharmacy programs in the United States adopted this software. ${ }^{22}$ It's use has expanded, as evidenced by more than 186 schools and colleges of pharmacy across 34 countries currently utilizing the program. ${ }^{23}$ As of June 2020, the program has highest adoption in the United States. ${ }^{24}$

Because of the higher numbers of schools of pharmacy using MyDispense in the United States, the program has been implemented in a wider range of courses including introductory and advanced pharmacy practice experiences (IPPEs and APPEs) than other countries. ${ }^{8,9,16,19,21,25}$

Most studies were focused on students' attitudes, perception, learning outcomes. No studies have been conducted among administrators to explore how MyDispense has been integrated into various courses of pharmacy programs in the United States. The objective of our study was to describe the use of MyDispense in pharmacy programs across the United States. This information, such as courses, skills, types of exercises, assessment features and more, may be useful for institutions considering adopting or expanding activities using MyDispense in their curricula.

\section{METHODS}

Researchers developed a 16-item self-administered electronic survey (Appendix 1) using Qualtrics software. ${ }^{26}$ The first section (9 questions) included institutional demographic information such as name of institution, year of MyDispense adoption, and number of courses that used the tool during the academic year 2019-2020. The second section (7 questions) asked about the use of MyDispense for each specific course at their institution. Pilot testing of the survey was conducted among two administrators to ensure clarity of questions. This study was classified as not regulated by the University of Michigan Health Sciences and Behavioral Sciences Institutional Review Board.

Researchers obtained a list of instructors in the US with MyDispense administrator accounts $(n=72$ administrators from 67 institutions). The administrators were typically key users of the MyDispense program at their institutions and they would be able to contact their colleagues to gather additional information about using MyDispense in their courses. All administrators were initially emailed to confirm if they were the correct person to respond to the survey. If an institution had more than one contact, clarification was obtained about which person should receive the survey. In addition, researchers provided a summary of the study questions and asked administrators to gather information from their colleagues who use MyDispense, if needed. A final contact list composed of 66 administrators (one for each institution) was created. 16 contacts were not affiliated with Doctor of Pharmacy Programs and therefore those institutions were excluded. A total of 50 pharmacy programs $(n=50 / 141,35 \%)$ were eligible to participate. ${ }^{27}$ Data collection occurred from July 1st to August 31st, 2020. During this time, four automatic reminders were set up to administrators who had not responded in order to encourage them to participate. The data was downloaded using MicroSoft Excel 2016. Descriptive statistics were used to analyze the results. All responses were included in our results.

\section{RESULTS}

A total of 36 responses (72\%) from schools or colleges of pharmacy across five regions, Midwest, Southeast, Northeast, West, and Southwest, were obtained (Table 1). Most were public institutions $(n=22,61 \%)$ and over one-third had implemented MyDispense in 2019 or 2020 ( $n=13,36 \%$ ). Institutional characteristics were different between responder and non-respondent institutions, for example, a majority of responder institutions were public $(\mathrm{n}=22,61 \%)$ and located in Midwest $(n=13,36 \%)$ whereas a majority of non-responder institutions were private $(n=8,57 \%)$ and located in Northeast $(n=5,36 \%)$ (Table 1). MyDispense was most commonly integrated into one $(n=12,46 \%)$ or two courses $(n=7$, $27 \%)$. About $50 \%$ of the respondents $(\mathrm{n}=13 / 24)$ reported that the use of MyDispense began or increased due to teaching during the severe acute respiratory syndrome coronavirus 2 (SARS-CoV-2) pandemic. 
MyDispense was utilized in 46 courses during the 2019-2020 academic year. Most courses ( $n=41,89 \%)$ were required courses and included only one professional year of student pharmacists $(n=43,93 \%)$. The remaining courses $(\mathrm{n}=3)$ were delivered to two cohorts of student pharmacists simultaneously (eg, P1 and P3 students) resulting in 49 course offerings (as the combined courses had different learning objectives for each cohort). One-half of courses ( $\mathrm{n}=25 / 49,51 \%)$ were delivered to first year student pharmacists, followed by second year $(n=10 / 49,20 \%)$, third year $(n=10 / 49,20 \%)$, and fourth year student pharmacists $(n=4 / 49,8 \%)$, respectively. The most common skills that student pharmacists practiced were medication dispensing, communication with patients, and drug-information skills (Table 2). Dispensing exercise were used in the most courses $(n=40)$, followed by validation $(n=29)$ and over-the-counter $(n=29)$. When validation exercises were included, there were more of these exercises $(n=19)$ than the dispensing $(n=16)$ or over-the-counter $(n=9)$ (Table 3). A total of 17 courses (37\%) used the assessment feature to grade MyDispense activities. Three-fourths of courses ( $n=35 / 46,76 \%)$ intended to use MyDispense during the upcoming academic year, while nine were unsure (20\%), and two intended to discontinue use (4\%). About $90 \%$ of the respondents $(n=32 / 36)$ were willing to share their information with instructors who use MyDispense in the United States and the MyDispense team at Monash University.

\section{DISCUSSION}

This study provides evidence to support the idea that virtual simulation, specifically via MyDispense, is increasingly being used among pharmacy programs in the United States. It is most frequently used in the first half of the curricula to provide student pharmacists practice with medication dispensing, communication with patients, utilizing drug information resources, applying pharmacy laws, and making recommendations about over-the-counter medications. This is similar to the use of MyDispense in Australia and United Kingdom where the program has been implemented in medication dispensing for first and second year student pharmacists. ${ }^{3,14,28}$ On the other hand, MyDispense were used in Malaysia and Saudi Arabia by more advanced student pharmacists (eg. fourth and fifth year) in their experiential practices/clerkships. ${ }^{29,30}$

The use of MyDispense has continued to grow during the COVID-19 pandemic, suggesting that attributes such as being free, web-based, customizable, and available to students $24 / 7$ are facilitators to utilization. ${ }^{21,29-31}$

The number of pharmacy schools that have adopted the MyDispense program have been continuously increasing since $2014 .{ }^{32}$ As of August 2020, more than one-third $(n=50 / 141,35 \%)$ of pharmacy schools across the US have gained administrative access to the program. ${ }^{27}$ This positive trend of the program adoption provides opportunities for collaboration, such as by creating common cases that can be used in similar courses in order to decrease faculty workload. As a result, instructors would have more time focusing on cases that are more complex/advanced or managing other crucial works. This may also decrease barriers to entry for new faculty interested in adopting this approach in their teaching. In South Africa, pharmacy schools expressed interested in using MyDispense leading to the South African MyDispense Forum being launched in May 2021. ${ }^{31}$ Our study provides insights into potential areas of focus for multiinstitutional and multi-country studies to evaluate effectiveness of student pharmacist learning. In particular, first- and second-year courses that target topics such as medication dispensing, drug information resources, and pharmacy law may align with existing utilization of the program while providing opportunities to shift from studying study perceptions and short-term educational gains in one course to longer-term educational gains. ${ }^{8,9,17,19-22,33,34}$ Instructors of schools of pharmacy in the United States and abroad can use this information to determine how and when MyDispense is a good supplementary learning resource.

The primary limitation of this study that not all Doctor of Pharmacy programs in the United States provided data. One administrator from each program was asked to provide information which may have resulted in estimated as opposed to actual numeric values reported. Institutions that did not respond or did not have contact information included on the initial list were not included. It is possible that institutions modified their approach to using MyDispense during the 20202021 academic year, particularly in light of the COVID-19 pandemic. Finally, data was not gathered about instructor attitudes or beliefs which may serve as facilitators or barriers to utilization of this program as we sought to collect utilization data from one key administrator per institution, as opposed to each individual instructor who used the tool. Future directions include identifying opportunities for sharing of exercises and assessment strategies as well as engaging in research to study student pharmacist learning.

\section{CONCLUSION}

Pharmacy program in the US are increasingly using MyDispense for students to practice critical patient care skills including medication dispensing, communication with patients, and drug-information skills. Instructors in the US most commonly used MyDispense in courses for first- and second-year student pharmacists to teach medication dispensing, communication with patients, utilizing drug information resources, applying pharmacy laws, and making recommendations about over-the-counter medications. 


\section{ACKNOWLEDGEMENTS}

The authors would like to acknowledge Keith Sewell, Keenan Beaumont, and Vivienne Mak from Monash University in Melbourne, Australia. The authors would also like to thank Abby Kahaleh, $\mathrm{PhD}$ for her feedback on this manuscript.

\section{REFERENCES}

1. Lucas C, Williams K, Bajorek B. Virtual pharmacy programs to prepare pharmacy students for community and hospital placements. Am J Pharm Educ. 2019;83(10):7011. doi:10.5688/ajpe7011

2. Seybert AL, Smithburger PL, Benedict NJ, Kobulinsky LR, Kane-Gill SL, Coons JC. Evidence for simulation in pharmacy education. J Am Coll Clin Pharm. 2019;2(6):686-692. doi:10.1002/jac5.1167

3. McDowell J, Styles K, Sewell K, et al. A Simulated Learning Environment for Teaching Medicine Dispensing Skills. Am J Pharm Educ. 2016;80(1):11. doi:10.5688/ajpe80111

4. Aebersold M. Simulation-based learning: No longer a novelty in undergraduate education. Online J Issues Nurs. 2018;23(2):1. doi:10.3912/OJIN.Vol23No02PPT39

5. Thisgaard M, Makransky G. Virtual Learning Simulations in High School: Effects on Cognitive and Noncognitive Outcomes and Implications on the Development of STEM Academic and Career Choice. Front Psychol. 2017;8:805. doi:10.3389/fpsyg.2017.00805

6. Veettil S, Rajiah K. Use of Simulation in Pharmacy Practice and Implementation in Undergraduate Pharmacy Curriculum in India. Int J Pharm Pharm Sci. 2016;8(7).

7. Mattsson S, Sjöström H-E, Englund C. Using a virtual tablet machine to improve student understanding of the complex processes involved in tablet manufacturing. Am J Pharm Educ. 2016;80(5):87. doi:10.5688/ajpe80587

8. Shin J, Tabatabai D, Boscardin C, Ferrone M, Brock T. Integration of a Community Pharmacy Simulation Program into a Therapeutics Course. Am J Pharm Educ. 2018;82(1):6189. doi:10.5688/ajpe6189

9. Mospan GA, Gillette C. Using MyDispense to simulate validation of controlled substance prescriptions in a pharmacy law course. Curr Pharm Teach Learn. 2020;12(2):193-202. doi:10.1016/j.cptl.2019.11.014

10. Eukel HN, Morrell B, Holmes SM, Kelsch MP. Simulation Design, Findings, and Call to Action for Managing Difficult Patient Encounters. Am J Pharm Educ. 2021;85(7):8327. doi:10.5688/ajpe8327

11. Lim AS, Lee SWH, Karunaratne N, Caliph S. Pharmacy Students' Perceptions and Performance on the Use of an Online Virtual Experience Tool for Practicing Objective Structured Clinical Examinations. Am J Pharm Educ. 2020;84(11):7920. doi:10.5688/ajpe7920

12. MyDispense. About-mydispense. https://info.mydispense.monash.edu/wp-content/uploads/2019/07/Aboutmydispense.pdf. Accessed December 12, 2021.

13. Fjortoft N, Gettig J, Verdone M. Response: We Need to Be Smarter Than Our Smartphones. Am J Pharm Educ. 2019;83(1):7477. doi:10.5688/ajpe7477

14. Nazar H, Lindsey L, Fletcher J, Rook L, Todd A, Husband A. Training student pharmacists in medication dispensing and checking within a high-fidelity clinical environment. Am J Pharm Educ. 2019;83(7):7106. doi:10.5688/ajpe7106

15. Ambroziak K, Ibrahim N, Marshall VD, Kelling SE. Virtual simulation to personalize student learning in a required pharmacy course. Curr Pharm Teach Learn. 2018;10(6):750-756. doi:10.1016/j.cptl.2018.03.017

16. Deneff M, Holle LM, Fitzgerald JM, Wheeler K. A Novel Approach to Pharmacy Practice Law Instruction. Pharmacy (Basel). 2021;9(2):75. doi:10.3390/pharmacy9020075

17. MyDispense. User guides. https://info.mydispense.monash.edu/support/user-guides/. Accessed December 12, 2021.

18. MyDispense. Instructors guide to admin. https://info.mydispense.monash.edu/wpcontent/uploads/2017/07/MyDispense-5.1-Instructors-guide-to-Admin.docx. Accessed December 12, 2021.

19. Tai MH, Rida N, Klein KC, et al. Impact of virtual simulation in self-care therapeutics course on introductory pharmacy practice experience self-care encounters. Curr Pharm Teach Learn. 2020;12(1):74-83. doi:10.1016/j.cptl.2019.10.015

20. Ferrone M, Kebodeaux C, Fitzgerald J, Holle L. Implementation of a virtual dispensing simulator to support US pharmacy education. Curr Pharm Teach Learn. 2017;9(4):511-520. doi:10.1016/j.cptl.2017.03.018

21. Kebodeaux C, Sewell K. Implementation of Community Pharmacy Dispensing Software (MyDispense) in an Advanced Pharmacy Practice Course. Am J Pharm Educ. 2016;80(5):S2 (p.70).

22. Costelloe MT. MyDispense: Lessons from Global Collaboration in Developing a Pharmacy Educational Simulation Tool. Innov Pharm. 2017;8(1). doi:10.24926/iip.v8i1.490 
23. Mak V, Fitzgerald J, Holle L, Vordenberg SE, Kebodeaux C. Meeting pharmacy educational outcomes through effective use of the virtual simulation MyDispense. Curr Pharm Teach Learn. 2021;13(7):739-742. doi:10.1016/j.cptl.2021.03.003

24. Mak V. "Responding to the Pandemic Together" Programme Episode 21 MyDispense: A virtual simulation to teach pharmacy students across the globe. https://www.fip.org/files/content/events/2020/FIP_COVID19_MyDispense_Monash_FINAL.pdf. Accessed December 12, 2021.

25. Johnson AE, Barrack J, Fitzgerald JM, Sobieraj DM, Holle LM. Integration of a Virtual Dispensing Simulator "MyDispense" in an Experiential Education Program to Prepare Students for Community Introductory Pharmacy Practice Experience. Pharmacy (Basel). 2021;9(1):48. doi:10.3390/pharmacy9010048

26. Qualtrics:. The Experience Management Platform. https://www.qualtrics.com/. Accessed December 12, 2021.

27. Accreditation Council for Pharmacy Education. Programs By Status. https://www.acpe-accredit.org/accreditedprograms-by-status/. Accessed December 12, 2021.

28. Chuang S, Grieve KL, Mak V. Analysis of Dispensing Errors Made by First-Year Pharmacy Students in a Virtual Dispensing Assessment. Pharmacy. 2021;9(1):65. doi:10.3390/pharmacy9010065

29. N.S.A Rahman, N.I.M. Nazar, M.H. Elnaem. Experiential learning in community pharmacy: Online and remote teaching experience in Malaysian higher education. https://pharmacyeducation.fip.org/pharmacyeducation/article/view/1137/947. Accessed December 12, 2021.

30. Al-Hindi Y, Mojally M. MyDispense impact in compensating summer field training course during COVID-19 pandemic. J Adv Pharm Educ Res. 2021;11(1):35-38.

31. Burton S. MyDispense: Taking pharmacy education into the future together. SA Pharm J. 2021;88(3):42-43.

32. MyDispense. Partner Institutions. https://info.mydispense.monash.edu/community/partner-institutions/. Accessed December 12, 2021.

33. Brown S, Kebodeaux C, Schadler A, Beamont K, \& Sewell K. Analysis of student performance outcomes using virtual dispensing exercises. Am J Pharm Educ, 2018;82(5):562. doi:10.5688/ajpe7158

34. Dameh M. A Report of Second Year Pharmacy Students' Experience after Using a Virtual Dispensing Program. $J$ Pharma Care Health Sys. 2015;S2(003). doi:10.4172/jpchs.1000-S2-003 


\begin{tabular}{lcc}
\hline & $\begin{array}{c}\text { Responded to Survey } \\
\text { n }(\boldsymbol{\%})\end{array}$ & $\begin{array}{c}\text { Did Not Respond to Survey } \\
\mathbf{n}(\boldsymbol{\%})\end{array}$ \\
\hline Region & & \\
Midwest & $13(36)$ & $2(14)$ \\
Northeast & $7(19)$ & $5(36)$ \\
Southeast & $10(28)$ & $1(7)$ \\
Southwest & $2(6)$ & $3(21)$ \\
West & $4(11)$ & $3(21)$ \\
Type of institution & & \\
Private & $14(39)$ & $8(57)$ \\
Public & $22(61)$ & $6(43)$ \\
Accreditation status & & $14(100)$ \\
Accredited & $35(97)$ & - \\
Year MyDispense was implemented & & - \\
2014 & $2(5.6)$ & - \\
2015 & $3(8.3)$ & - \\
2016 & $5(13.9)$ & - \\
2017 & $1(2.8)$ & - \\
2018 & $2(5.6)$ & - \\
2019 & $6(16.7)$ & - \\
2020 & $7(19.4)$ & $14(100)$ \\
Not implemented & $9(25)$ & - \\
Information not provided & $1(2.8)$ & \\
\hline
\end{tabular}

Table 2. Number of Courses Teaching Specific Skills Using MyDispense by Student Year

Number of Courses n (\%)

Skills Students Practiced Using MyDispense

Medication dispensing

Communication with patients

Drug-information skills

Pharmacy law

Over-the-counter

Communication with healthcare professionals

Therapeutic decision making

Dosage forms

Calculations related to patient assessment

Ethical decision making

Interpreting laboratory values and diagnostic tests

Dietary supplements

Non-sterile compounding

Other (MyDispense Version 6 feature)

${ }^{a}$ Courses that enrolled students in multiple ye

Table 3. Number and Types of MyDispense Exercises Utilized within Each Course ${ }^{\mathrm{a}}$

\begin{tabular}{|c|c|c|c|}
\hline \multirow[b]{2}{*}{ Type of Exercise (Number of Courses Utilizing Exercise Type) } & \multicolumn{3}{|c|}{ Average number of exercises (range) } \\
\hline & Required & Optional & Total \\
\hline Dispensing/prescription $(\mathrm{n}=40)$ & $10(2-31)$ & $6(1-21)$ & 16 \\
\hline Validation/checking station $(n=29)$ & $11(1-61)$ & $8(1-24)$ & 19 \\
\hline Over-the-counter $(n=29)$ & $7(1-30)$ & $2(1-4)$ & 9 \\
\hline
\end{tabular}

\footnotetext{
${ }^{\mathrm{a}}$ Among each course that uses this type of exercise.
} 
Appendix 1. MyDispense Key Administrator Survey

We would like to know how MyDispense is used at your institution.

What year was MyDispense initially used at your institution?

$2013-2020$ [drop down]

I do not know

I have not implemented MyDispense into my course, but I'm interested in it.

How many courses at your institution used MyDispense during the academic year 2019-2020? (If you have more than 9 courses, please select the top 9 courses where MyDispense is used.)

0 - 9 [drop down]

These following questions are for each individual course in MyDispense has been used during the 2019-2020 academic year.

What is the course name?

What year students participate in this course? Select all that apply. [P1, P2, P3, P4]

Is this course required or an elective? [Required, Elective]

What topic or skills do students practice using MyDispense in this course? Select all that apply.

Calculations related to patient assessment

Communication with healthcare professionals

Communication with patients

Dietary supplements

Dosage forms

Drug-information skills

Ethical decision making

Interpreting laboratory values and diagnostic tests

Medication dispensing

Non-sterile compounding

Over-the-counter (OTC)/Self-Care

Pharmacy law

Sterile compounding

Therapeutic decision making

Other, please provide

How many of each of the following types of exercises are in this course? Please fill out the total number of individual exercises. For example, if one validation exercise includes three prescriptions, count this as three. If there are no exercises of this type, type 0.

\begin{tabular}{|c|l|l|}
\hline Types of exercises & Required & Optional \\
\hline $\begin{array}{c}\text { Dispensing/ } \\
\text { Prescription }\end{array}$ & & \\
\hline $\begin{array}{c}\text { Validation/ Checking } \\
\text { station }\end{array}$ & & \\
\hline $\begin{array}{c}\text { Over-the-counter/ Self- } \\
\text { care }\end{array}$ & & \\
\hline
\end{tabular}

Is the assessment feature in MyDispense used to grade activities or exams? Mark "yes" or "no" for each type of exercise. If there are no exercises of this type, mark "no."

\begin{tabular}{|c|l|l|}
\hline \multirow{2}{*}{ Types of exercises } & \multicolumn{2}{|l|}{ Assessment features } \\
\cline { 2 - 3 } & Yes & No \\
\hline $\begin{array}{c}\text { Dispensing/ } \\
\text { Prescription }\end{array}$ & & \\
\hline $\begin{array}{c}\text { Validation/ Checking } \\
\text { station }\end{array}$ & & \\
\hline $\begin{array}{c}\text { Over-the-counter/ Self- } \\
\text { care }\end{array}$ & & \\
\hline
\end{tabular}

Will MyDispense be used in this course next year? [Yes, No, Not sure]

We would like to learn more about your institution.

Where is your institute located? [drop down list of states]

Is your institution public or private? [Public, Private]

How did COVID-19 impact the use of MyDispense at your institution? 
Increased use of MyDispense

No change in use of MyDispense

Decreased use of MyDispense

Started use MyDispense 